\title{
Interpreting the line-profile variations of subdwarf B pulsators: the case of PG 1336-018 (NY Virginis) ${ }^{\star}$
}

\author{
M. Vučković ${ }^{1}$, R. H. Østensen ${ }^{1}$, C. Aerts ${ }^{1,2}$, J. H. Telting ${ }^{3}$, U. Heber ${ }^{4}$, and R. Oreiro ${ }^{1}$ \\ ${ }^{1}$ Institute for Astronomy, K. U. Leuven, Leuven, Belgium \\ e-mail: maja.vuckovic@ster.kuleuven.be \\ 2 Department of Astrophysics, Radboud University, Nijmegen, The Netherlands \\ 3 Nordic Optical Telescope, Santa Cruz de La Palma, Spain \\ 4 Dr. Remeis-Sternwarte, Universität Erlangen-Nürnberg, Bamberg, Germany
}

Received 09 May 2009 / Accepted 25 June 2009

\section{ABSTRACT}

\begin{abstract}
Aims. We analyze the high-resolution time-resolved VLT/UVES spectra of PG 1336-018, a rapidly pulsating subdwarf B (sdB) primary in a close orbit with an M5 companion, with the aim to detect the pulsational signal of the primary in line-profile variations. Methods. After removing the dominant radial-velocity component inherent to the orbital motion and taking only out-of-eclipse data we computed cross-correlation functions ( $\mathrm{CCFs}$ ) for each individual spectrum and assumed these to approximate the average line profile. We computed predictions of line-profile variations for pulsating sdB stars and present their diagnostic value for mode identification.

Results. We detect the pulsation mode at $5435 \mu \mathrm{Hz}$ in the line-profile variations of the CCFs which allows us to characterize the detected pulsation mode of PG 1336-018.

Conclusions. We model the pulsational perturbations of synthetic spectra for a star with physical parameters like PG 1336-018 and confront them with the observed spectra. We find that low order Balmer lines can be used as diagnostic tool to identify pulsation modes. A detailed line-profile analysis of the perturbed time-series spectra excludes the $\ell=|m|=(3,3),(1,0),(2,1)$ and $(2,0)$ modes, but does not allow a unique mode identification among the radial or sectoral dipole or quadrupole modes.
\end{abstract}

Key words. subdwarfs - binaries: eclipsing - stars: variables: general - line: profiles - stars: oscillations stars: individual: PG 1336-018

\section{Introduction}

Subdwarf B (sdB) stars are core-helium burning stars (Heber et al. 1984) at the blue end of the horizontal branch. While the future evolution of sdB stars is quite certain, the exact prior evolutionary paths remain to be fully resolved. For a detailed review on hot subdwarfs we refer to the recent review by Heber (2009). The discovery that some sdB stars pulsate (Kilkenny et al. 1997) allows us to probe their inner structure via asteroseismology which can help us find definite answers about their prior evolution. The first step in seismic modeling, after identifying the frequencies of pulsation, is to identify the pulsation modes. One of the ways to identity the modes of pulsation is through the analysis of the line-profile variations.

There have been several radial velocity (RV) studies using low-resolution spectroscopy of rapidly pulsating $\mathrm{sdB}$ stars (now termed V361 Hya stars, but also known as sdBV or EC14026 stars, Kilkenny 2007). The first time-resolved spectroscopy study was done by Jeffery \& Pollacco (2000) on PB 8783 and KPD 1929+4401, followed by extensive studies on PG $1605+072$ by Woolf et al. (2002), O'Toole et al. (2000, 2002, 2003a, 2005) and Tillich et al. (2007), on PG 1325+101 by Telting \& Østensen (2004), on Balloon 090100001 by Telting \& Østensen (2006) and Østensen et al. (2008) and most recently on PG $1219+534$ by Reed et al. (2009). These low-resolution

* Based on observations from the European Southern Observatory, Chile. Program ID: 075.D-0174. spectroscopy studies were mainly focused on measuring the RV and equivalent width variations, as well as characterization of the pulsational effective temperature and gravity variations.

The field of sdB star asteroseismology is booming, thanks to the gathering of a huge amount of data sets (both photometry and low-resolution spectroscopy) on a large number of pulsating sdB stars (for an overview see Østensen 2009). The lack of time-series of high-resolution sdB spectra, however, is apparent. Considering the requirements for the successful interpretation of line-profile variations: good temporal resolution, high signal-to-noise ratio $(S / N)$, and high resolving power (Aerts \& Eyer 2000; Mantegazza 2000), this lack of high-resolution timeresolved V361 Hya spectra is not surprising at all. The pulsational characteristics of V361 Hya stars are indeed poorly suited for classical line-profile variation studies. The short pulsation periods of V361 Hya stars, of the order of a few minutes, require short exposure times (below $1 \mathrm{~min}$ ) to avoid smearing the pulsational signal during the cycle (Aerts et al. 2008). Moreover, the faintness of the V361 Hya stars precludes the required $S / N$ for any line-profile variation study. Adding to this the fact that V361 Hya stars are low amplitude multiperiodic pulsators (photometric amplitudes are of the order of a few millimagnitudes) with rich and closely spaced frequency spectra, we can easily conclude that, even for the biggest telescopes with sophisticated detectors, they pose a considerable challenge.

The first high-resolution $(R=20000)$ spectroscopic timeseries on a V361 Hya star was assembled for PG 1605+072 
$\left(m_{\mathrm{B}}=12.8\right)$ with the FUSE satellite (Kuassivi et al. 2005) with the aim of measuring the projected broadening of the photospheric lines. They derive the line-profile variation diagram by phase folding the spectra into the eight phase intervals of the main pulsation period $(P=480 \mathrm{~s})$ and co-adding the four strongest photospheric lines detected (a N III doublet and three $\mathrm{S}$ IV lines). From this they derive $v \sin i$ of $21 \pm 9 \mathrm{~km} \mathrm{~s}^{-1}$ and report that the observed phase opposition between the maximum radius and the maximum velocity is consistent with adiabatic pulsations.

In Vučković et al. (2007, hereafter Paper I), we presented the first ground based high-resolution $(R=46890)$ spectroscopic time-series on a V361 Hya star, using the Ultraviolet and Visual Échelle Spectrograph (UVES) at Very Large Telescope (VLT). The aim of that work was to determine the orbital solution and mass of the components of the eclipsing binary PG 1336-018 $\left(m_{\mathrm{B}}=13.6\right)$ by combining high-speed multicolor VLT/ULTRACAM ${ }^{1}$ lightcurves (about two full orbits) and the RV curve obtained from high-resolution time-series of about $400 \mathrm{VLT} / \mathrm{UVES}$ spectra. We found three solutions of equal statistical significance due to the large parameter space and correlations between parameters, two of which are consistent with standard binary evolution models ( $\mathrm{Hu}$ et al. 2007). One of the favored solutions of Paper I with the mass of the sdB primary of $0.466 M_{\odot}$ was recently found by Charpinet et al. (2008) who arrive at the same mass by independent asteroseismic modeling through frequency matching of the observed oscillation frequencies found by Kilkenny et al. (2003).

The very first study of pulsation-mode identification based on high-resolution $(R=25000)$ spectroscopy data, aiming at identifying the dominant pulsation mode in the star, has been reported only recently by Telting et al. (2008) for the high amplitude sdB pulsator $\left(m_{\mathrm{B}}=11.8\right)$ Balloon 090100001. By phase folding the spectra onto the dominant mode $(P=356 \mathrm{~s})$ and combining the information of 56 narrow absorption metal lines in each spectrum they create cross-correlation functions (CCFs) which are of sufficient $S / N$ for a mode-identification analysis. From the model fits to the pulsational line-profile variations in the CCF they rule out quadrupole modes $(\ell=2)$ and show that the radial pulsation $(\ell=0)$ and a dipole pulsation $(\ell=1)$ are both consistent with the data, putting further constraints on the pulsation characteristic of the main mode of Balloon 090100001. In particular, the amplitude of the dominant mode was found to be $15 \mathrm{~km} \mathrm{~s}^{-1}$.

In this paper we further exploit the VLT/UVES observations of PG 1336-018 described in Paper I with the aim to study the pulsational signal of the primary in line-profile variations. This is the first application of a line-profile variation study on a V361 Hya star with low photometric amplitudes ( $<8 \mathrm{mmag})$, no dominant frequency and a dense and narrow frequency spectrum (28 frequencies were found in a $5000 \mu \mathrm{Hz}$ range, see Table 4 of Kilkenny et al. 2003). All previous time-series spectroscopy studies, including the recent line-profile analysis by Telting et al. (2008), were done on V361 Hya outliers with exceptionally high amplitudes (O'Toole et al. 2000, 2002, 2003a, 2005; Woolf et al. 2002; Telting \& Østensen 2004, 2006; Tillich et al. 2007).

\section{Observational diagnostics}

We presented in detail the data on PG 1336-018 in our previous work (Paper I). The data reduction using MIDAS routines was sufficient for the orbital analysis but did not give good enough

${ }^{1}$ ULTRACAM is an ultra-fast, triple-beam CCD camera.

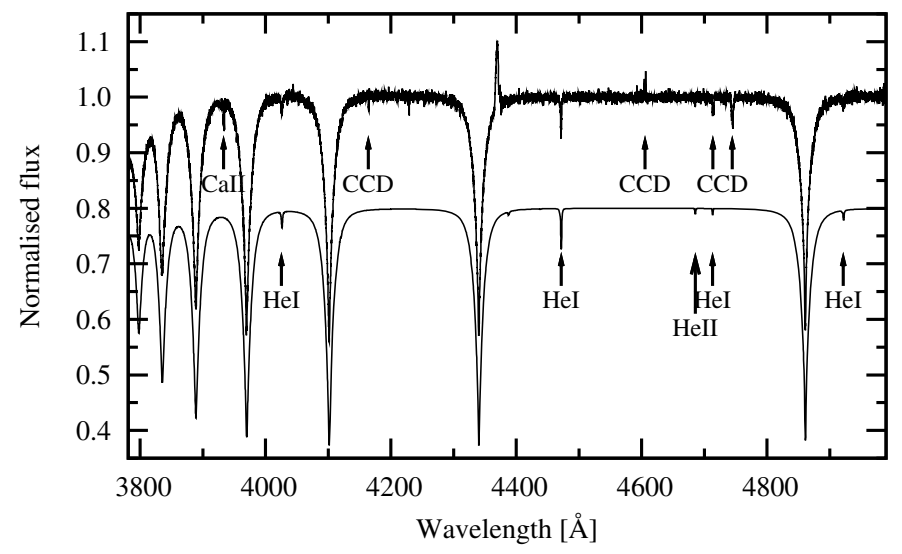

Fig. 1. The mean spectrum of PG 1336-018 produced by combining all the VLT/UVES spectra after shifting according to the orbital RV solution from Paper I, together with the model spectrum used as a template for cross-correlation. The features marked with "CCD" result from the defects of the CCD chip.

results for the line-profile analysis (see Fig. 2 of Paper I and text explaining the data quality). Here the raw data have been completely re-reduced using IRAF $^{2}$ tasks. The new mean orbitcorrected spectrum as shown in Fig. 1 is an improvement on that of Paper I (Fig. 2). Nevertheless, the spike in the red wing of $\mathrm{H}_{\gamma}$ is still present, being an artifact caused by the dual amplifier readout used in the ultrafast mode. The feature around $3933 \AA$ in the mean spectrum is the interstellar Ca II line which is artificially broadened due to the shifting of the individual spectra to correct for the orbital motion. The features marked with "CCD" result from the defects of the CCD chip and are also broadened due to the orbit correction. The mean spectrum is completely dominated by the broad Balmer lines, with a few He I lines and one He II line, as marked in Fig. 1. No metal lines are detected.

For the orbit correction, we use the orbital solution found in Paper I. To check for any pulsation signature in the data, we calculated the radial velocities of the new orbit corrected spectra in the same way as described in Paper I, i.e. using the molly ${ }^{3}$ software package. The measured RVs were subjected to periodogram analysis with Period04 (Lenz \& Breger 2004). A peak in the periodogram was found at the frequency $(5434 \pm 6) \mu \mathrm{Hz}$ with an amplitude of $(3.5 \pm 0.6) \mathrm{km} \mathrm{s}^{-1}$ and a signal-to-noise ratio $(S / N)$ of 3.3. Even though the $S / N$ is quite low, the frequency found is considered to be present in the data because it is the same one as found in all other independent data sets ever taken on PG 1336-018 (Kilkenny et al. 1998; Reed et al. 2000; Kilkenny et al. 2003) including our ULTRACAM data set (Paper I). Encouraged by detecting the pulsation signature in the spectra, we exploit the data further to investigate the pulsational information content in them.

\subsection{Cross-correlation profiles}

The noise level in the individual spectra $(S / N$ of about 10 , see Paper I) does not permit us to analyse the line-profile variations in each spectrum. The successful mode identification based on

\footnotetext{
2 IRAF is distributed by the National Optical Astronomy Observatories, operated by the Association of Universities for Research in Astronomy, Inc., under cooperative agreement with the National Science Foundation.

${ }^{3}$ http://deneb.astro.warwick.ac.uk/phsaap/software/ molly/html/IN \discretionary-DEX.html
} 
line-profile variations requires typically a $S / N$ ratio above 150 (Aerts et al. 2008). To increase the $S / N$ in the profiles, we have to combine the information contained in various line profiles, via e.g. a cross-correlation technique. The $S / N$ in the CCFs depends on the number of spectral lines used for the cross-correlation, $n$, and their corresponding $S / N$ in the form (Hekker et al. 2006):

$S / N_{\text {cross-cor }}=\sqrt{n\left\langle(S / N)^{2}\right\rangle}$.

CCFs have been used before to study the character of oscillation modes, see e.g. Mathias \& Aerts (1996) for the $p$ modes in the $\delta$ Scuti star $20 \mathrm{CVn}$, Hekker et al. (2006) for solar-like oscillations in red giants and De Cat et al. (2006) for the $g$ modes in $\gamma$ Doradus stars.

CCFs have been considered already in the study of sdB stars, mostly to measure the RV variations due to the pulsations, e.g. in the stars PB 8783 and KPD 1929+4401 (Jeffery \& Pollacco 2000) and PG $1605+072$ (Woolf et al. 2002) to state a few. Recently, Telting et al. (2008) used a cross-correlation technique on Balloon 090100001 to combine the information from many narrow metal lines into a single high $S / N$ profile in order to investigate the signature of its oscillation modes. Unfortunately, the spectrum of PG 1336-018 is devoid of any metal lines, due to the high rotational broadening $\left(v \sin i\right.$ of $74.2 \mathrm{~km} \mathrm{~s}^{-1}$ see Paper I) smearing out the lines. Even in the orbit corrected mean spectrum (Fig. 1) there are only Balmer lines and few weak He I lines which are hidden in the noise in the individual spectra. Unable to use any narrow metal lines for cross-correlation, we have to rely on the broad hydrogen and weak helium lines present in our spectra to get the most information possible.

For this purpose we first used the mean orbit corrected spectrum shown in the top panel of Fig. 1 as the template for crosscorrelation on the orbit corrected out-of-eclipse spectra. The resulting CCFs, however, were very noisy. As we identified three He I (4921 $\AA, 4471 \AA$ and $4026 \AA$ ) lines in the mean spectrum, we attempted at using just those He I lines to compute the CCFs. We used the central wavelengths of these three He I lines to create a template spectrum consisting of delta functions, rotationally broadened with the observed $v \sin i$. This method did not give satisfactory results (CCFs were too noisy) as we retrieve only the $4471 \AA$ line in each individual spectrum and hence the $S / N$ in the CCFs does not improve.

To increase the $S / N$ in the CCFs we made a model spectrum using the $\mathrm{H}+\mathrm{He}$ LTE models of Heber et al. (2000) shown in Fig. 1 that reproduced the observed spectrum the best. Using this model spectrum as a template (from red end up to $\mathrm{H}_{\zeta}=\mathrm{H}_{8}$ ) for the cross-correlation turned out to be the optimal method for our particular data set. Fig. 2 shows an individual PG 1336-018 spectrum around $\mathrm{H}_{\beta}$ with the model spectrum used as a crosscorrelation template. The CCFs for each individual spectrum are presented in Fig. 3. Since each CCF represents an average line profile within the region of the spectrum analysed, for the case of PG 1336-018, the average line profile is dominated by Balmer lines. The intrinsic profiles of Balmer lines and consequently the CCFs have significantly broadened wings due to a linear Stark effect and therefore strongly deviate from a Gaussian profile.

\section{Search for the oscillations in the various line diagnostics}

In general, there are two ways to describe line-profile variability: as variations in velocity space (e.g. represented as velocity moments) or as variations in intensity as a function of position in the line profiles. Accordingly, there are two methods available for

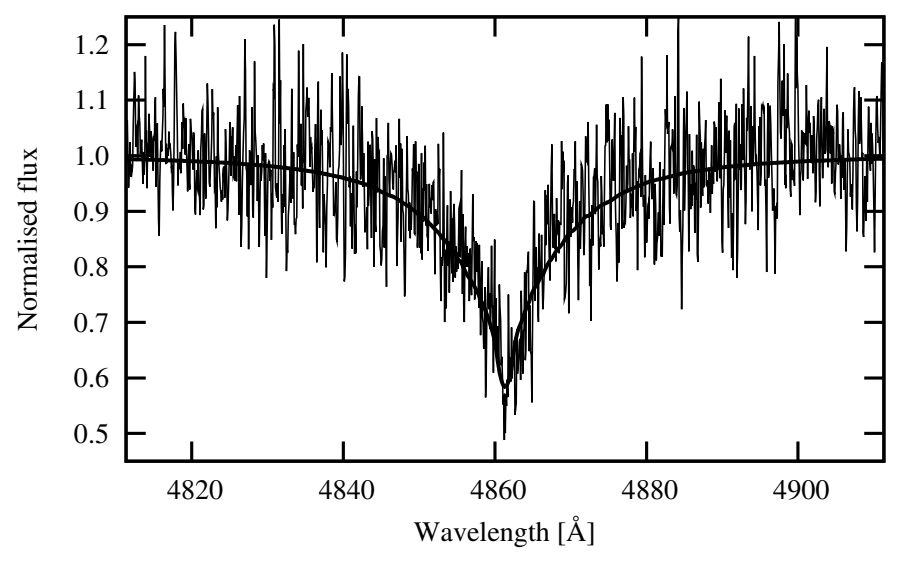

Fig. 2. Observed individual spectrum around $\mathrm{H}_{\beta}$ overplotted with the synthetic spectrum used as a cross-correlation template.

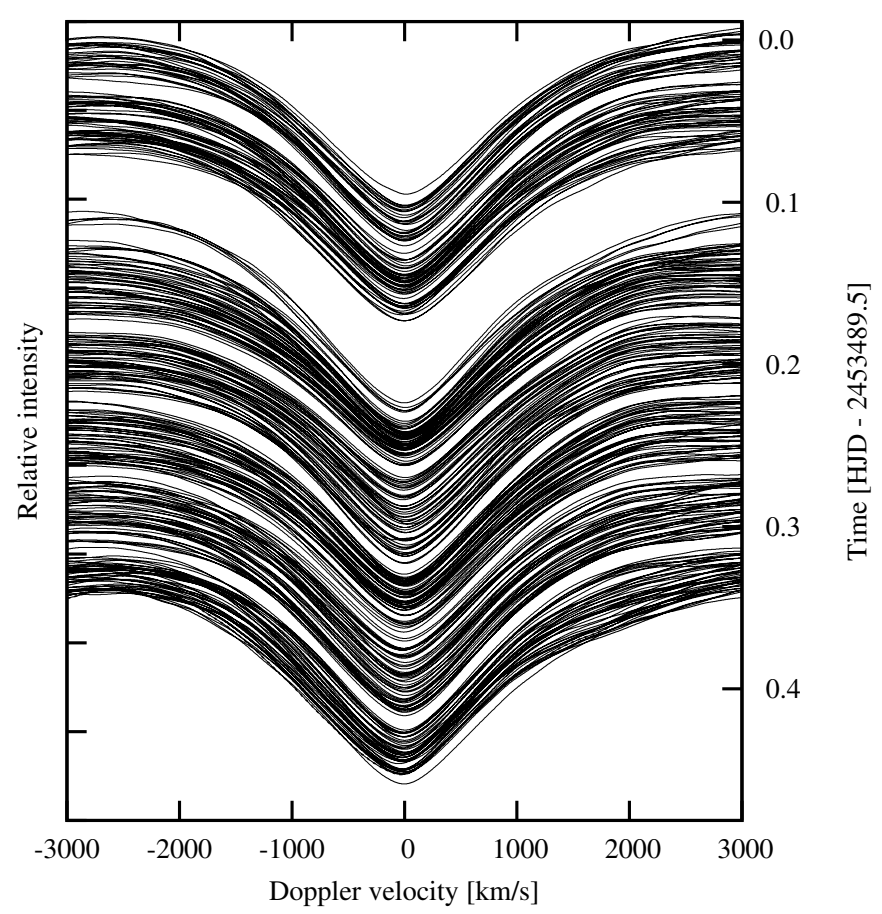

Fig. 3. Cross-correlation profiles of our out-of-eclipse PG 1336-018 spectra. The tick marks on the left axis indicate a difference of $10 \%$ in relative intensity units. Each profile has been shifted downward by the time since midnight UT on the night of observations, as indicated on the right axis.

analysing the pulsational characteristics of line profiles: the moment method (Briquet \& Aerts 2003, and references therein) and Fourier parameter fit method (FPF, Zima 2006). Both methods assume a Gaussian intrinsic line profile. The moment method is based on the time variations of the first few moments of a line profile and it is optimally suited for slow rotators. The FPF method relies on the rotational broadening of a line profile and delivers good and reliable results for $v \sin i>20 \mathrm{~km} \mathrm{~s}^{-1}$ for the resolution we are dealing with here.

\subsection{Frequency analysis for various line diagnostics}

We search for periodicities in the CCF by analysing the line moments and intensity variations on the individual velocity bins across the CCF using the freely available software package 


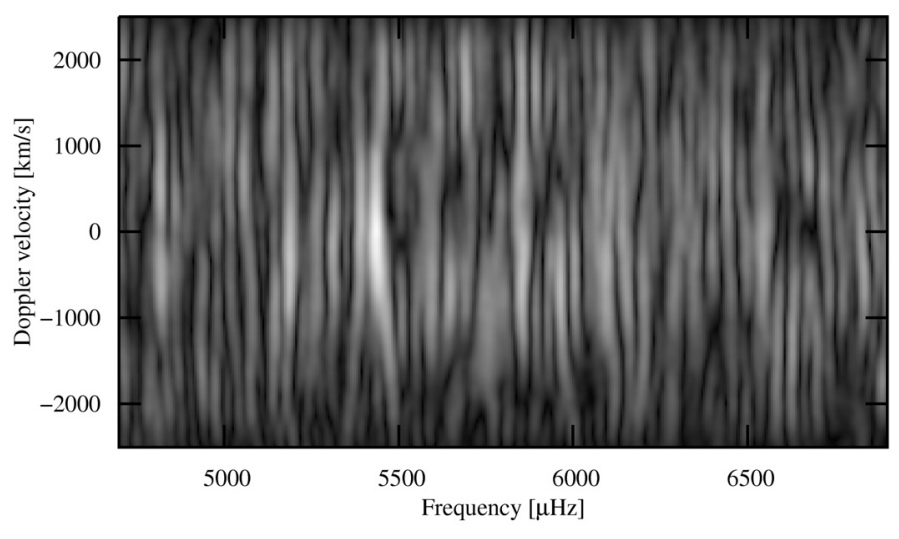

Fig. 4. The Fourier amplitude spectrum of the all out-of-eclipse PG 1336-018 spectra. The gray scale is given in continuum units with the range $0-0.002$ (black to white).

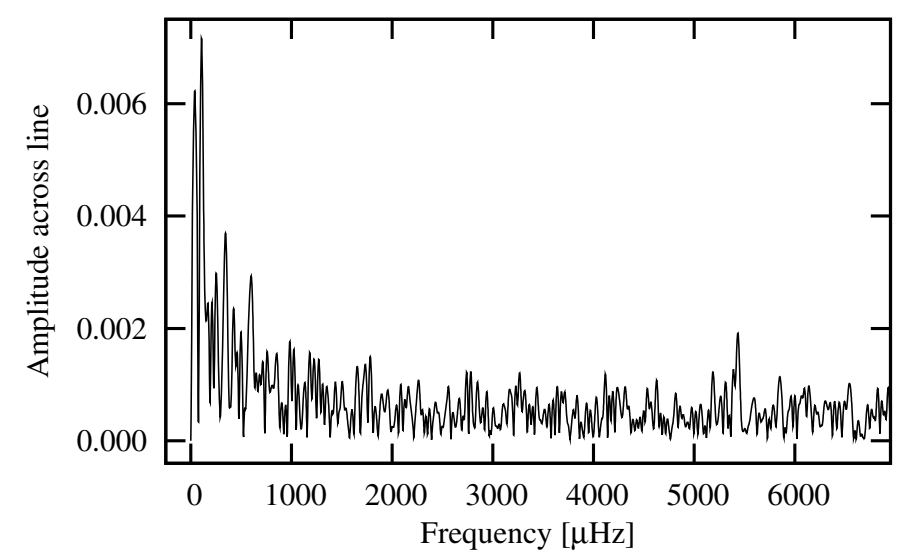

Fig. 5. Fourier spectrum of the intensity variations across the crosscorrelation profiles over the $[-100,100] \mathrm{km} \mathrm{s}^{-1}$ dispersion range (pixelby-pixel method).

FAMIAS $^{4}$ (Zima 2008). To analyse the amplitude distribution across the profile we calculate the Fourier amplitude spectrum of the time-series of the cross-correlation profiles. For each wavelength (velocity) bin, the amplitude as a function of frequency is plotted in Fig. 4, with the amplitude given as a gray value in continuum units. Though the Fourier amplitude spectrum is quite noisy, there is a clear variability concentrated around a narrow core of the CCF in the frequency domain where the peak in the $\mathrm{RV}$ is seen (at $5435 \mu \mathrm{Hz}$ ).

We then compute a Fourier spectrum which is the mean of all Fourier spectra across the narrow dispersion range around the core where the main variation is seen (pixel-by-pixel method). The resulting Fourier spectrum over the $[-100,100] \mathrm{km} \mathrm{s}^{-1}$ dispersion range for the whole frequency domain up to the Nyquist frequency is shown in Fig. 5. A frequency at $5435 \mu \mathrm{Hz}$ with amplitude of 0.0019 (in continuum units) and with a $S / N$ of 4.2 is detected.

A second approach to search for periodicities was carried out by computing the first three velocity moments of the CCFs (for a definition, see Aerts et al. 1992) and performing a frequency search on the time-series of moments over the same dispersion range. The Fourier transformations of the equivalent width (zero moment) and first three moments are shown in the lower

\footnotetext{
4 The mode identification software package FAMIAS developed in the framework of the FP6 European Coordination Action HELAS (http://www . helas-eu.org/).
}

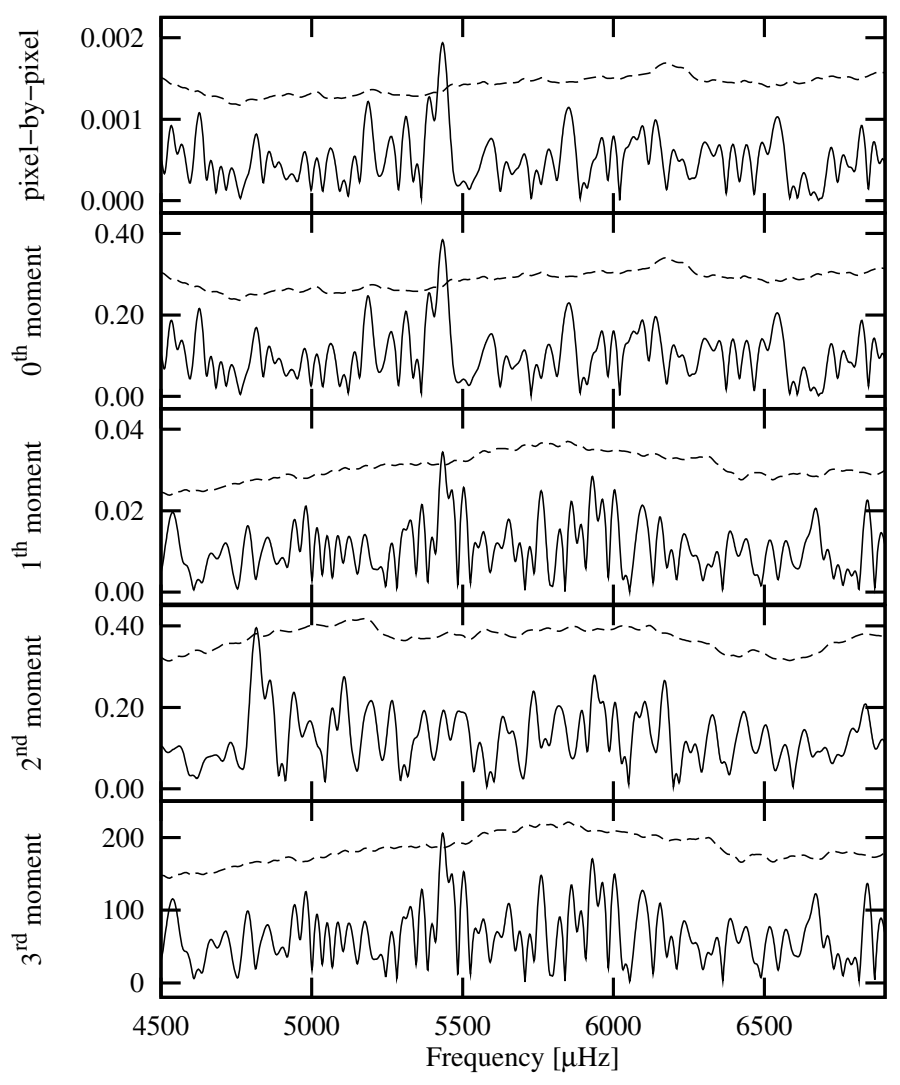

Fig. 6. Fourier analysis of the cross-correlation profiles over the $[-100,100] \mathrm{km} \mathrm{s}^{-1}$ dispersion range. The frequency domain of the main pulsations detected in the photometry is plotted together with the $3 \sigma_{\text {noise }}$ threshold (dashed line). The upper panel shows the Fourier analysis of the intensity variations across the profile (pixel-by-pixel method). The next panels show the Fourier analysis of the moments: zero, first, second and third (as marked).

panels in Fig. 6 together with the $3 \sigma_{\text {noise }}$ threshold. The frequency at $5435 \mu \mathrm{Hz}$ is detected in both types of diagnostics (FPF and moments), but with different significance. The highest amplitude of the detected frequency is found by the pixel-by-pixel method and in the equivalent width variations with equal $S / N$ of 4.2, while the first and the third moment (representing the centroid velocity and the line skewness, respectively) detect the same frequency with equal significance of $S / N=3.3$. The frequency is not detected in the second moment (which measures the line width changes) of the CCF, which hints toward nonaxisymmetric modes (Aerts et al. 1992).

There are two main noticeable features while comparing the variabilities: the similarity in the distribution of the frequencies of the pixel-by-pixel variations and equivalent width variations, and the similarity in shape of the variations of the first and third moments. While the latter one is not surprising considering the fact that first and third moment are not independent, the former one is indeed striking. They are both due to the narrow dispersion range over which the summation of the FTs was done. While the FWHM of the average CCF is $\sim 1700 \mathrm{~km} \mathrm{~s}^{-1}$ the variability is detected only in the narrow core of the CCFs. Since we are analysing only the core of the CCFs and not the whole crosscorrelation profiles it is not surprising that the equivalent width variations of the core are dominated by the pixel-to-pixel variations. 


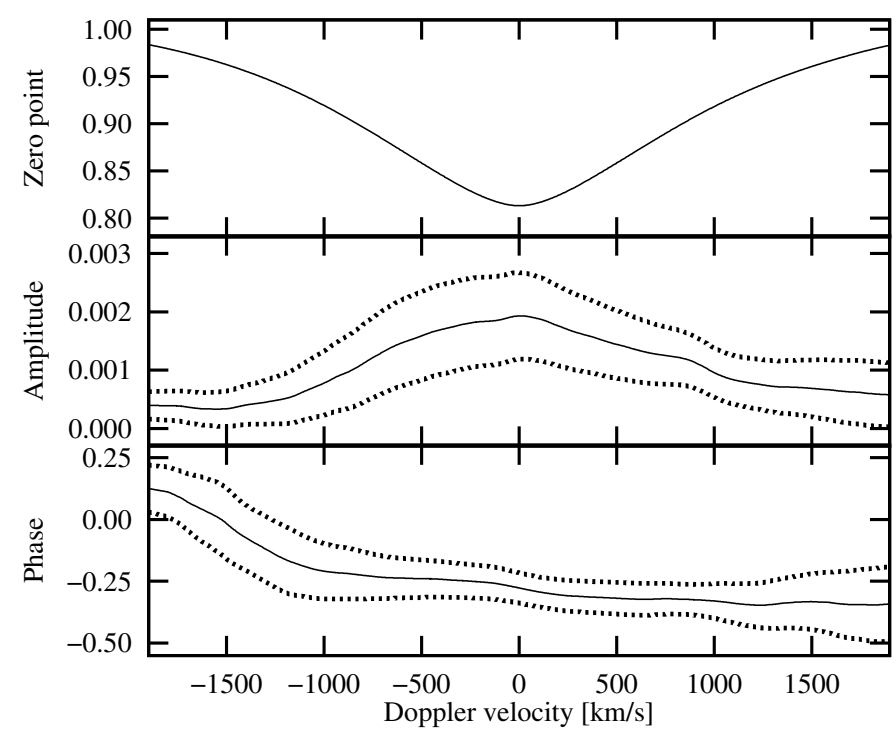

Fig. 7. The Fourier parameters across the cross-correlation profiles of the detected pulsation frequency. The average profile (termed zero point in FAMIAS) is shown at the top, the observed amplitude distribution across the profile (solid line) in the middle together with the error band (dotted) and the observed phase distribution (solid line) in the bottom with the error band (dotted).

\subsection{The mode character}

Although this is the first time the pulsational signal has been detected in the line-profile variations of PG 1336-018, the aim of any asteroseismological study is to identify the pulsation mode, besides detecting its frequency. Therefore we proceed further with analysing the characteristics of the detected frequency. As PG 1336-018 is a fast rotator FPF should be the most suitable method for mode identification. The FPF method makes use of the fact that the zero point $\left(Z_{0}\right)$, amplitude $\left(A_{0}\right)$ and phase $\left(P_{0}\right)$ across the line profile depend on the $(\ell, m)$-values of the associated pulsation modes. By comparing the theoretical values of zero profile, amplitude and phase (ZAP) with the observed values, one can, in principle, determine the degree $\ell$ and azimuthal order $m$ of a pulsation mode. In the FPF method, the equivalent width variations of the line profile due to local temperature variations at the stellar surface can be accounted for.

For the detected pulsation frequency and each dispersion bin across the CCF profile, we compute a non-linear least-squares fit of sinusoids. This delivers the observational values of ZAP as a function of the position in the line profile. The Fourier parameters across the $\mathrm{CCF}$ profile with their associated errors, as calculated by FAMIAS are presented in Fig. 7.

An immediately apparent feature is the absence of a double peaked behavior of the amplitude across the profile and the flat phase, i.e. the absence of a phase jump at the line center. This implies that the mode we detect is not an axisymmetric $(m=0)$ $p$-mode pulsation (Schrijvers et al. 1997). This argument is also supported by considering the effect of the inclination: for the stars seen almost equator-on the detection of sectoral modes $(\ell=|m|)$ is favored. The orbital inclination of PG 1336-018 is well constrained to $i=(80.67 \pm 0.08)^{\circ}$ (see Paper I). Here we assume that the symmetry axis of pulsation has the same inclination, which is a valid approach for such a close orbit. For a discussion on the alignment of the axis, see Reed \& Whole Earth Telescope Xcov 21 and 23 Collaborations (2006).

The short time baseline of our data set hampers the increase in $S / N$ by phase folding. Phase folding onto the pulsation frequency increases the $S / N$ in the case there is a dominant (or at least well resolved) mode, as recently shown by Telting et al. (2008) on Balloon 090100001. We have tried to phase fold the data onto the detected frequency, but that did not increase the $S / N$, most likely due to the fact that the phase-folded data are contaminated by neighboring unresolved frequencies. Within the frequency resolution of our data set (about $30 \mu \mathrm{Hz}$ ), there are at least three closely spaced frequencies detected (Kilkenny et al. 2003) all with similar photometric amplitudes $\sim 2$ mmag (see discussion in Sect. 4.2 of Paper I). Therefore the phase-folding technique is not applicable to our data set.

\section{Computation of synthetic line-profile variations}

To interpret the line-profile variations in our PG 1336-018 spectra we have to model the various pulsational modes and compare them with the observed features. We generate a grid of timeseries theoretical profiles using the BRUCE and KYLIE routines (Townsend 1997) with the timings of our data set and the detected frequency. BRUCE takes as input a set of physical stellar parameters and a pulsation mode with a given $(\ell, m)$, pulsation period and velocity amplitude. From these parameters it produces a list of surface elements with a specific temperature, gravity, RV, area and viewing angle. KYLIE then integrates these elements into an effective observed spectrum for each point in the pulsation cycle. To accomplish this, KYLIE requires a threedimensional grid of specific intensity spectra covering the required range in temperature and gravity as well as viewing angle. The computation of the grid of specific intensity spectra is also a two step process. We started by using a grid of fully metal line blanketed LTE model atmospheres (Heber et al. 2000), which were plane parallel and chemically homogeneous and consisted of hydrogen, helium and solar metal abundances. From these we used Lemke's version of the LINFOR spectrum synthesis program (Lemke 1997) to compute the specific intensity spectra required by KYLIE.

A note of caution regarding the metallicity is required at this point. The chemical composition of sdB stars is known to be highly peculiar, showing large deficiencies for the lighter elements (e.g. C and $\mathrm{Si}$ ) on the one hand, and strong overabundances of heavy elements as deduced from UV and FUV spectra on the other (see O'Toole \& Heber 2006; Blanchette et al. 2008), while iron is found to be approximately solar in most sdBs. The line blocking of UV iron lines is responsible for most of the line blanketing affecting the temperature-density stratification of the model atmosphere. Because the heavy elements, such as the iron group, can be analysed from UV spectra only, we have no means to determine their abundances in PG 1336-018. Hence we adopted solar values for them in the model atmosphere calculations. However, synthetic spectra calculated with solar abundances display metal lines (mostly of light elements) that are far too strong when compared to the observed spectrum, even when taking the strong rotational broadening into account. In order to produce synthetic spectra that resemble the mean observed optical spectrum as well as possible, we included metal lines at abundances depleted by a factor 100 relative to solar in the LINFOR spectral synthesis.

The stellar parameters used for modelling the line-profile variations are taken from the orbit solution of Paper I and are summarized in Table 1 . The inclination angle of PG 1336-018 is constrained from the orbit solution and therefore this parameter was fixed at $i=80.67^{\circ}$. Keeping in mind that this mode is detected in photometry we computed a grid of synthetic time-series 
Table 1. Stellar parameters used to model the line-profile variations using the spectral-synthesis codes BRUCE and KYLIE (Townsend 1997).

\begin{tabular}{ll}
\hline \hline Parameter & Value \\
\hline Equatorial rotational velocity $\left(\mathrm{km} \mathrm{s}^{-1}\right)$ & 75 \\
Inclination angle $\left(^{\circ}\right)$ & 80.67 \\
Polar radius $\left(R_{\odot}\right)$ & 0.15 \\
Polar temperature $(\mathrm{K})$ & 31300 \\
Polar gravity $\left(\mathrm{cm} \mathrm{s}^{-2}\right)$ & 5.77 \\
Pulsation period & $(\mathrm{s})$ \\
\hline
\end{tabular}

${ }^{a}$ The period detected in our VLT/UVES data set and in all photometric campaigns on PG 1336-018 (see Paper I).

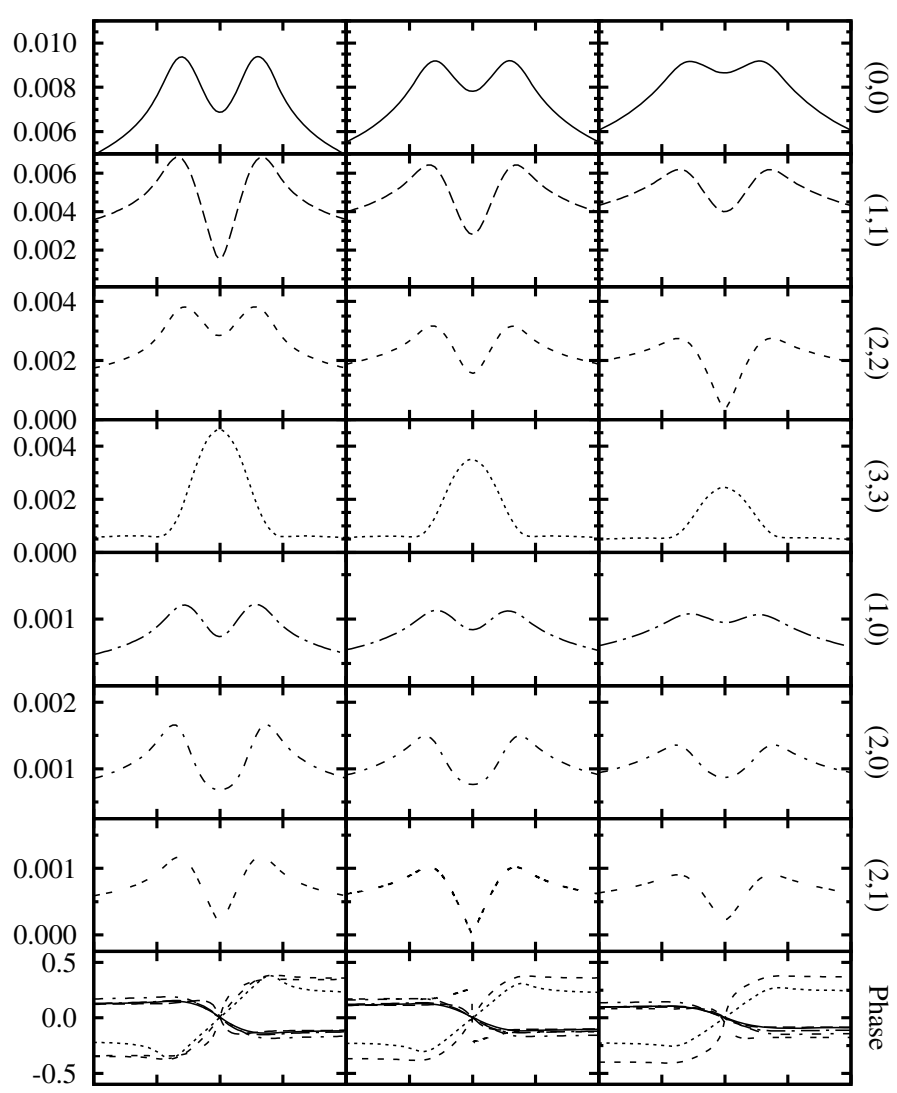

Fig. 8. The amplitude and phase (bottom panel) distribution across the profiles for the core of the synthetic Balmer lines $\mathrm{H}_{\beta}$ (left column), $\mathrm{H}_{\gamma}$ (middle column) and $\mathrm{H}_{\delta}$ (right column) for retrograde and axisymmetric modes. The amplitude across the profile (in continuum units) as a function of Doppler velocity (ranging from -200 to $200 \mathrm{~km} \mathrm{~s}^{-1}$ ) of the modes $(\ell, m)$ as marked. The phase is given in units of $\pi$.

for modes $\ell \leq 3$ and for velocity amplitudes $\mathrm{VA}=2,4,6,8$ and $10 \mathrm{~km} \mathrm{~s}^{-1}$.

The analysis of O'Toole et al. (2003a) and Telting \& $\emptyset$ stensen (2004) on the line-profile variations on PG 1605+072 and PG $1325+101$, respectively, finds that the surface gravity and temperature variations vary with the same period as the RV variation. Furthermore, Telting \& Østensen (2004) explain the variations seen in the profiles of Balmer lines as pulsational surface gravity, surface temperature and RV variations (see their Fig. 9). As we are studying the line-profile variations of Balmer lines we have included the gravity and temperature perturbations together with the velocity perturbations. We use a non-adiabatic factor of 0.5 in the Buta \& Smith (1979) equation in BRUCE (see the BRUCE manual for an explanation) as found to be required for Balloon 090100001 by Østensen et al. (2008).

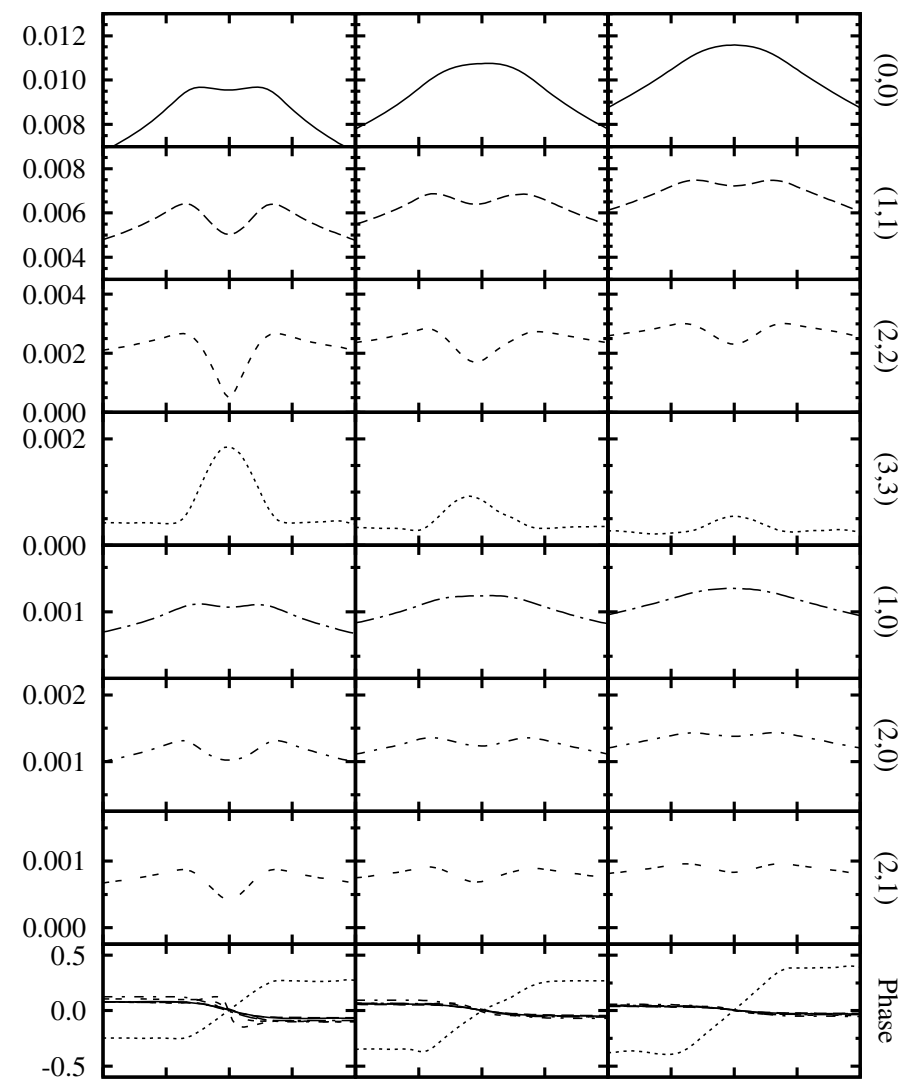

Fig. 9. The same as Fig. 8 for $\mathrm{H}_{\epsilon}$ (left column), $\mathrm{H}_{\zeta}$ (middle column) and $\mathrm{H}_{\eta}$ (right column).

We analyse the behavior of each Balmer line from $\mathrm{H}_{\beta}$ to $\mathrm{H}_{\eta}$ across the synthetic profile for $\ell \leq 3$. The variations in both amplitude and phase are clearly visible but only in the narrow core $\left(< \pm 200 \mathrm{~km} \mathrm{~s}^{-1}\right)$. From the grid of model calculations, we select a VA of $10 \mathrm{~km} \mathrm{~s}^{-1}$ to illustrate the Balmer line behavior. Figures 8-11 show the amplitude and phase diagnostics for the synthetic Balmer lines per each $\ell \leq 3$ mode. As expected for such a high inclination angle, modes with $m \neq \ell$ suffer from significant cancellation effects, and sectoral modes $(\ell=|m|)$ are favoured. This, however, is the case if we assume that all modes have the same intrinsic amplitude. The intrinsic amplitudes of $(\ell \neq m)$ modes would have to be much higher in order to reach the same amplitudes across the profiles as sectoral modes for this inclination angle, see Sect. 5.

The most important point to note is that the retrograde and prograde modes of the same $(\ell,|m|)$ are not the mirror images of each other, see Figs. 8 and 10 for example. This is due to the way the velocity fields and temperature perturbations generate the line-profile variability. The temperature perturbations cause changes in the continuum intensity and changes to the line-profile equivalent width. The surface element with the increased temperature will form an absorption feature in the line profile of the disk integrated spectrum. If the equivalent width of the given line profile is increasing with increasing temperature this absorption feature will be enhanced, whilst if the equivalent width is decreasing with increasing temperature the absorption feature will be reduced and could become an emission feature. Equivalent width variations of Balmer lines are described by a nonlinear combination of temperature and surface gravity (O'Toole et al. 2003a). For hot stars such as sdBs, the change of equivalent width with temperature is negative, so that the equivalent width becomes smaller with increasing temperature and the 


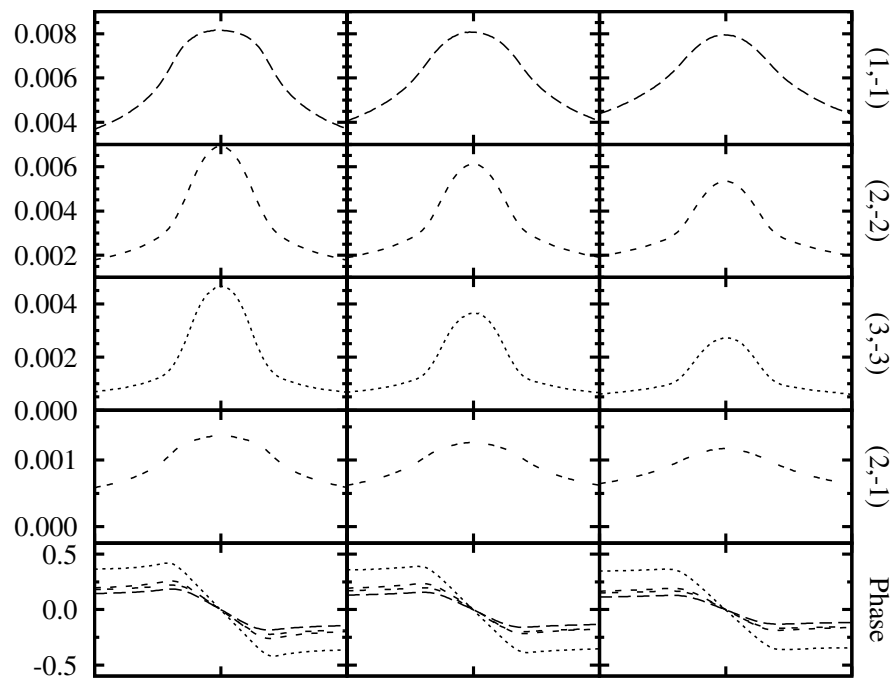

Fig. 10. The same as Fig. 8 for prograde modes for $\mathrm{H}_{\beta}$ (left column), $\mathrm{H}_{\gamma}$ (middle column) and $\mathrm{H}_{\delta}$ (right column). The amplitude across the profile (in continuum units)as a function of Doppler velocity (ranging from -200 to $200 \mathrm{~km} \mathrm{~s}^{-1}$ ) of the modes $(\ell, m)$ as marked. The phase (bottom) is given in units of $\pi$.

Balmer lines are becoming shallower as the temperature is increasing and deeper as the temperature is decreasing.

Maximum temperature in retrograde mode line profile occurs when the velocity perturbed line profile is at its deepest, and minimum temperature when the velocity perturbed line profile is at its shallowest point. The up-down motion is damped so that the sideways motion dominates, producing a central dip in the amplitude profile. For the prograde modes, however, the minimum temperature in the line profile occurs when the velocity perturbed line profile is at its deepest point and the maximum temperature when the velocity perturbed line profile is at its shallowest point, hence reinforcing the up-down motion of the core of the line profile over the sideways motion, creating a single peaked amplitude profile.

The overall amplitude of the line profile is increasing with increasing order along the Balmer series. The rise in the amplitude across the $\mathrm{H}_{\zeta}$ and $\mathrm{H}_{\eta}$ profiles is due to their higher sensitivity to the temperature and gravity effects, and the fact that there is no actual continuum in the higher order Balmer region (see Fig. 1) as the lines are overlapping. O'Toole et al. (2003b) have also found a strong dependence of line index amplitude on Balmer line order, with high order Balmer line amplitudes up to 10 times larger than $\mathrm{H}_{\beta}$ due to the fact that the line index may not only be dependent on temperature but also on surface gravity.

The Balmer lines clearly show a different amplitude and phase variation across the profile for different modes of oscillation and can therefore in principle be used in an attempt to identify the mode. Each Balmer line shows a phase shift at the center. The phase distribution across all Balmer lines for $\ell=0$ and $\ell=|m|=1$ is similar while the phase difference between the blue and red line wings for these modes, $\Delta \Psi_{0,0}$ and $\Delta \Psi_{1,1}$, is decreasing with increasing Balmer order from $0.3 \pi$ for $\mathrm{H}_{\beta}$ to $0.1 \pi$ for $\mathrm{H}_{\eta}$. The phase distribution for $\ell=m=2$ is different from $\ell=-m=2$ mode. The phase difference for $\mathrm{H}_{\beta}, \mathrm{H}_{\gamma}$ and $\mathrm{H}_{\delta}$ for $\ell=m=2$ is higher than for $\ell=-m=2: \Delta \Psi_{2,2}$ of $0.7 \pi$ compared to $\Delta \Psi_{2,-2}$ of $0.4 \pi$, and decreasing from $\mathrm{H}_{\epsilon}$ to $\mathrm{H}_{\eta}$ where it blends with $\Delta \Psi_{0,0}$ and $\Delta \Psi_{1,1}$. The $\ell=|m|=3$ mode is the most distinguishable among the modes considered with respect to both amplitude and phase distribution. The amplitude,

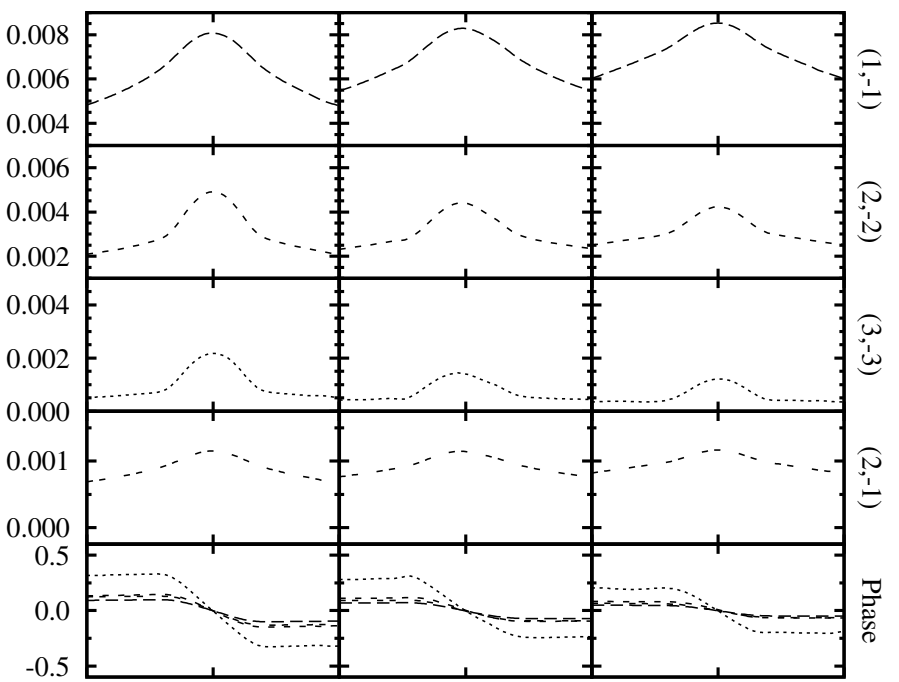

Fig. 11. The same as Fig. 10 for $\mathrm{H}_{\epsilon}$ (left column), $\mathrm{H}_{\zeta}$ (middle column) and $\mathrm{H}_{\eta}$ (right column).

even though decreasing with the Balmer order, has a peak at the line center as opposed to the central dip for the $\ell=m=0,1$ and 2 modes for both retrograde and prograde case. The phase difference of the $\ell=m=3$ mode is the only one that increases with the Balmer order from $\Delta \Psi_{3,3}$ of $0.5 \pi$ for $\mathrm{H}_{\beta}$ to $0.8 \pi$ for $\mathrm{H}_{\eta}$, while for $\ell=-m=3$, the phase difference is decreasing with Balmer order from $0.7 \pi$ for $\mathrm{H}_{\beta}$ to $0.4 \pi$ for $\mathrm{H}_{\eta}$.

To visualise the amplitude and phase behavior of each Balmer line we subtracted the time averaged synthetic spectrum from each individual one. The time-series of subsequent residual spectra in gray scale are plotted in Fig. 12 for all $\ell$ and $m$ modes considered above, for the region of variability around each Balmer line.

For such a high intrinsic width of Balmer lines (much greater than the rotational broadening of PG 1336-018) the variability of the surface elements is smeared out over a large part of the profile. Interestingly, there is still a distinct variability in cores of Balmer lines which makes them usable for spectral mode identification provided the spectra have enough $S / N$. Due to the fact that there is no actual continuum in the higher order Balmer region and their higher sensitivity to surface gravity variations $\mathrm{H}_{\zeta}$ and $\mathrm{H}_{\eta}$, although carrying valuable information on gravity variations, are difficult for the interpretation with the current modeidentification techniques.

\section{Confrontation between simulated predictions and observed diagnostics}

To explore how the line-profile variations are preserved in $\mathrm{CCF}$ we calculate the CCF of the synthetic spectra in the same way as for the data. Using the same model spectrum (from $\mathrm{H}_{\beta}$ to $\mathrm{H}_{\zeta}$ ) as a template, we cross-correlate the synthetic time-series for each pulsation mode with $\ell=(0,1,2$ and 3$)$ and $-\ell \leq m \leq \ell$. The CCFs of the simulated time-series do all preserve the frequency of the oscillation that is stretched up to $1000 \mathrm{~km} \mathrm{~s}^{-1}$ around the CCF core. The amplitude and phase across the CCF profile, however, are not straightforward to interpret. The CCF profiles of the intrinsically very broad Balmer lines are smeared out and lose the sensibility of detecting the small variations in the narrow core of the lines. The amplitude across the CCF profiles does not recover the narrow $\left( \pm 100 \mathrm{~km} \mathrm{~s}^{-1}\right)$ central dip for $(\ell, m)=(0,0)$ and $(1,1)$ modes due to the fact that there is a 

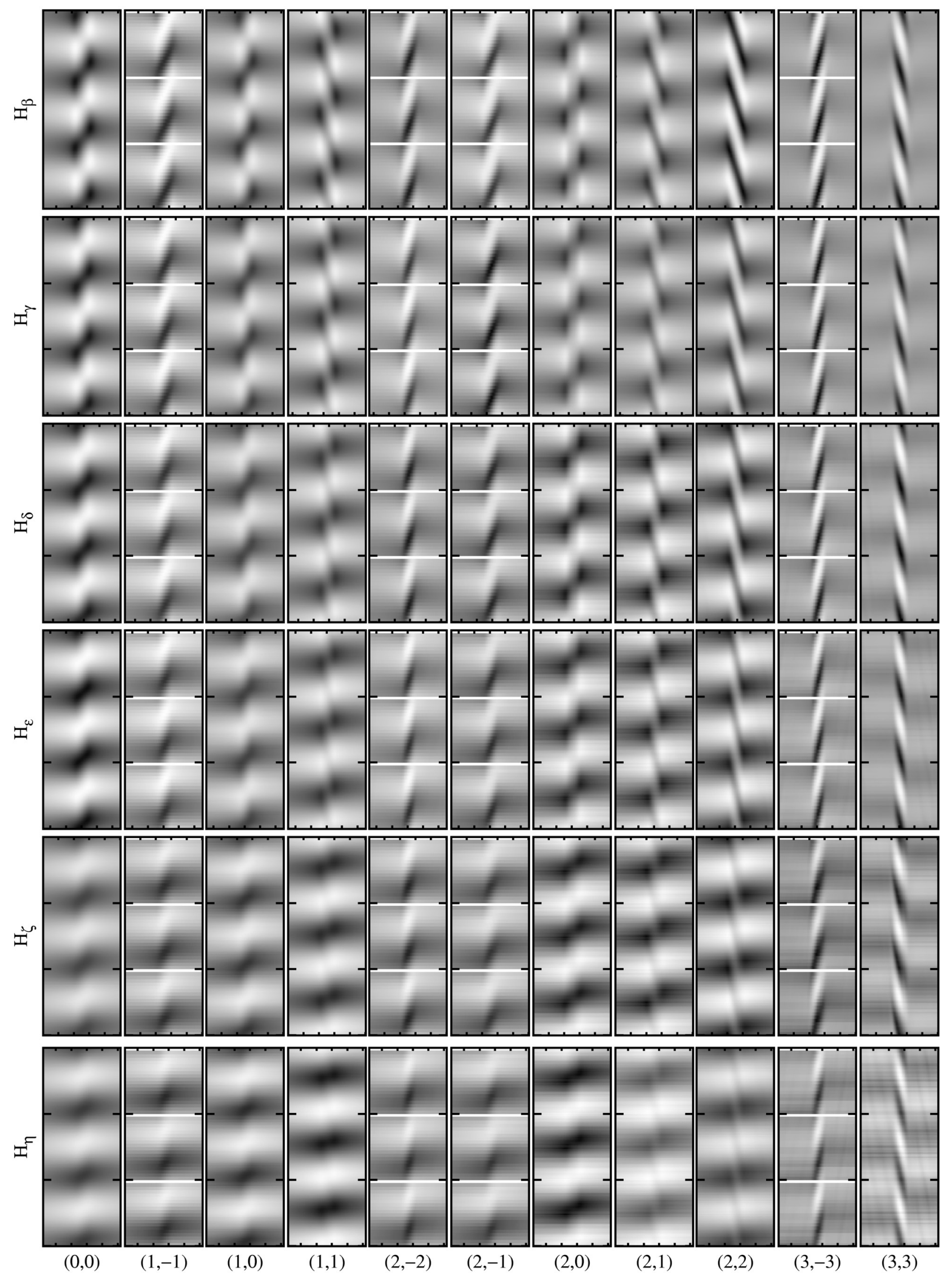

Fig. 12. Three pulsation cycles of residual (mean subtracted) spectra shown as gray scale images for the core of the synthetic Balmer lines $\mathrm{H}_{\beta}$ to $\mathrm{H}_{\eta}$ (top to bottom) for the $(\ell, m)$ combinations as marked below the bottom panel. Black indicates intensities less than the average and white indicates intensities greater than average. The separation between horizontal ticks is $2 \AA$ and between vertical one pulsation phase. 
high overall amplitude in the wings (increasing toward the core) of the lines for these modes. The amplitude across the CCF profiles for $(\ell, m)=(2,2)$ and $(3,3)$ modes recovers the dip and the bump in the core of the lines, respectively, due to the lower amplitude in the wings for these modes. The phase across the CCF profiles is smeared out as well, but it does preserve the overall trends.

While the cross-correlation technique is used to smear out the noise it also smears the signal in such a broad crosscorrelation template. After examining the amplitude and phase variations of different Balmer lines, we conclude that crosscorrelation of several Balmer lines may not preserve the lineprofile variations in a way traceable for the mode identification as different Balmer lines do not have the same line-profile behavior for the same mode of oscillation (see Figs. 8-11).

To test if different Balmer lines can be combined to achieve better $S / N$ and still preserve their amplitude and phase distribution across the combined profile, we selected lines that show similar line-profile behavior namely $\mathrm{H}_{\beta}, \mathrm{H}_{\gamma}, \mathrm{H}_{\delta}$ and $\mathrm{H}_{\epsilon}$ and calculated their average line profile. The average line profile preserved the amplitude and phase distribution of the original lines excellently. We have tried the same with the observed spectra both for all the out-of-eclipse and for the spectra taken during the secondary eclipse but as the averaging of four lines gives only a factor of 2 in $S / N$ we still fail to detect the pulsation signal during secondary eclipse.

Even though the pulsation signal is obviously highly smeared out in the broad CCFs we confront the CCFs of the observed spectra with those of the synthetic spectra which have been manipulated in the same way as the data, to check if any feature emerges. Figure 13 shows the amplitude (top) and phase (bottom) distribution of the CCFs of the data and CCFs the synthetic time-series for the modes with reasonable amplitudes to fit the observed range (see the caption). Clearly the errors on the amplitude and phase distribution across the CCFs are too high to let us attempt any unique mode identification. Note that the velocity amplitude of the $(\ell,|m|)=(3,3),(1,0),(2,1)$ and $(2,0)$ modes would have to be unrealistically high, VA $>100 \mathrm{~km} \mathrm{~s}^{-1}$ for $(\ell,|m|)=(3,3)$ and VA $\geq 30 \mathrm{~km} \mathrm{~s}^{-1}$ for the $(\ell,|m|)=(1,0)$, $(2,1)$ and $(2,0)$ in order to reach the amplitude of the data and therefore are not plotted. Interestingly, the phase of the CCFs of the data set fits rather well the phase distribution of the $(\ell,|m|)=(0,0),(1,1)$ and $(2,2)$ modes in the narrow region where the variability is seen. Due to the large errors (gray area in Fig. 13) we cannot make any further conclusions. The phase distribution of CCFs of $\ell=0$ and $\ell=|m|=1$ modes is indistinguishable. While the amplitude and phase distribution can in principle distinguish between $\ell=|m|=0,1$ and $\ell=|m|=2$ modes, the amplitude and phase distribution of our data set is too noisy to exclude any of the $\ell=|m|=0,1$ or 2 modes.

\section{Conclusions}

Due to the low signal in the individual spectra we were not able to analyse the line-profile variations of the individual lines in the time-series of PG 1336-018 spectra but instead we analyse the variations of the CCFs that approximate the average line for each spectrum. The lack of metal lines in PG 1336-018 spectrum forced us to work with CCFs of broad Balmer lines which smear out the pulsation signal. Nevertheless, we detected one pulsational frequency in the CCFs of PG 1336-018 in all line diagnostics. This is the first time a pulsational signal has been detected in high-resolution spectra of PG 1336-018. The detected frequency has equal significance in the pixel-by-pixel method
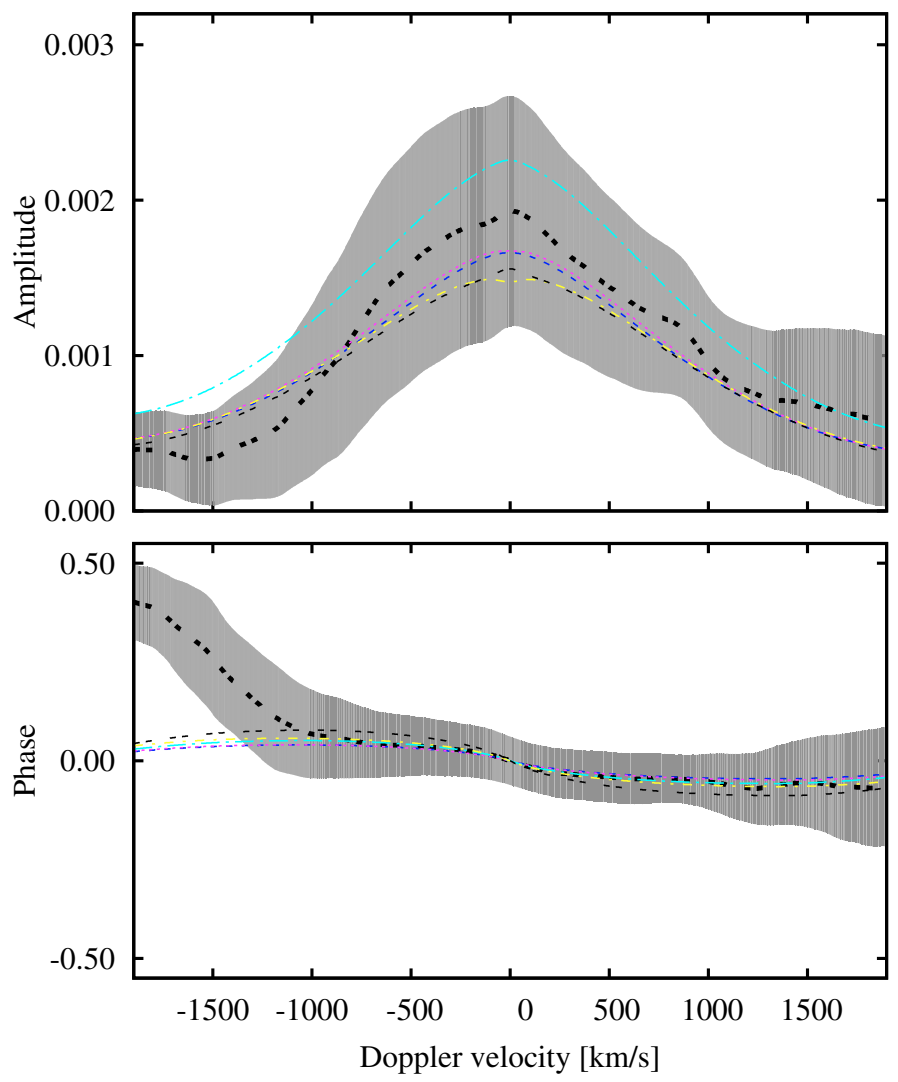

Fig. 13. The amplitude (upper) and phase (bottom) distribution of the CCFs of the data (thick double dashed line) with errors (gray area) overplotted with the amplitude and phase distribution of the CCFs of the synthetic spectra for modes with realistic amplitudes. The amplitude is given in continuum units and phase in units of $\pi$. The radial mode with $\mathrm{VA}=4 \mathrm{~km} \mathrm{~s}^{-1}$ is shown (thin dashed line), the $(\ell, m)=(1,1)$ with $\mathrm{VA}=6 \mathrm{~km} \mathrm{~s}^{-1}$ (dotted line), the $(\ell, m)=(1,-1)$ with $\mathrm{VA}=8 \mathrm{~km} \mathrm{~s}^{-1}$ (long dash-dot line), while the $(\ell,|m|)=(2,2)$ modes only for VA $\geq$ $14 \mathrm{~km} \mathrm{~s}^{-1}$ reach the observed range, given as thin short dash-dot for $(\ell, m)=(2,2)$ and thin double dashed line for $(\ell, m)=(2,-2)$. The amplitudes of the $(\ell, m)=(1,0),(2,1)$ and $(2,0)$ modes would have to be $\geq 30 \mathrm{~km} \mathrm{~s}^{-1}$ to reach the observed amplitudes, while the $(\ell, m)=(3,3)$ mode only with VA $>100 \mathrm{~km} \mathrm{~s}^{-1}$ fits into the observed range.

and in the variation of the equivalent width, while the same frequency with somewhat lower amplitude is detected in the analysis of the first and third velocity moments. Phase folding onto the detected pulsation frequency to increase the $S / N$ is not suitable for the case of our short time base spectra of PG 1336-018 as it does not have an isolated high amplitude frequency.

Although suitable for the case of PG 1336-018 with respect to the high rotational velocity, we were not able to use the FPF spectroscopic mode-identification technique as implemented in the software tool FAMIAS on the CCF of PG 1336-018 as this method assumes Gaussian intrinsic line profiles and therefore is not suitable for the lines whose intrinsic profile deviates from a Gaussian.

However, from the overall qualitative amplitude and phase distribution analysis of the observed versus synthetic CCFs we conclude that the mode detected in the PG 1336-018 spectra is not an $(\ell,|m|)=(3,3),(1,0),(2,1)$ or $(2,0)$ mode. While the amplitude and phase distribution can in principle distinguish between $\ell=|m|=0,1$ and $\ell=|m|=2$ modes, in our data they are too noisy to exclude any of the radial, sectoral dipole or quadrupole modes. It would be interesting to compare the seismic solutions for these options in a $\chi^{2}$ sense, but we cannot 
deduce this information from the study of Charpinet et al. (2008). Our most important conclusion is that the low order Balmer lines can be used as diagnostic tool to identify pulsation modes, provided that each individual spectrum has a sufficiently high $S / N$ ratio (at least 200), as we have shown with our simulated time-series.

Acknowledgements. M. V. acknowledges a Ph.D. scholarship from the Research Council of Leuven University. M. V., C. A. and R. Ø. are supported by the Research Council of Leuven University, through grant GOA/2008/04. M. V. is thankful to Wolfgang Zima and Fabien Carrier for always being there to discuss the cross-correlation technique and for letting us use their codes.

\section{References}

Aerts, C., \& Eyer, L. 2000, in Delta Scuti and Related Stars, ed. M. Breger, \& M. Montgomery, PASPC, 210, 113

Aerts, C., de Pauw, M., \& Waelkens, C. 1992, A\&A, 266, 294

Aerts, C., Hekker, S., Desmet, M., et al. 2008, in Precision Spectroscopy in Astrophysics, ed. N. C. Santos, L. Pasquini, A. C. M. Correia, \& M. Romaniello, 161

Blanchette, J.-P., Chayer, P., Wesemael, F., et al. 2008, ApJ, 678, 1329

Briquet, M., \& Aerts, C. 2003, A\&A, 398, 687

Buta, R. J., \& Smith, M. A. 1979, ApJ, 232, 213

Charpinet, S., van Grootel, V., Reese, D., et al. 2008, A\&A, 489, 377

De Cat, P., Eyer, L., Cuypers, J., et al. 2006, A\&A, 449, 281

Heber, U. 2009, ARA\&A, 47, in press

Heber, U., Hunger, K., Jonas, G., \& Kudritzki, R. P. 1984, A\&A, 130, 119

Heber, U., Reid, I. N., \& Werner, K. 2000, A\&A, 363, 198

Hekker, S., Aerts, C., de Ridder, J., \& Carrier, F. 2006, A\&A, 458, 931

Hu, H., Nelemans, G., Østensen, R., et al. 2007, A\&A, 473, 569

Jeffery, C. S., \& Pollacco, D. 2000, MNRAS, 318, 974

Kilkenny, D. 2007, Commun. Asteroseismol., 150, 234

Kilkenny, D., Koen, C., O’Donoghue, D., \& Stobie, R. S. 1997, MNRAS, 285, 640
Kilkenny, D., O’Donoghue, D., Koen, C., Lynas-Gray, A. E., \& van Wyk, F. 1998, MNRAS, 296, 329

Kilkenny, D., Reed, M. D., O'Donoghue, D., et al. 2003, MNRAS, 345, 834

Kuassivi, Bonanno, A., \& Ferlet, R. 2005, A\&A, 442, 1015

Lemke, M. 1997, A\&AS, 122, 285

Lenz, P., \& Breger, M. 2004, in The A-Star Puzzle, ed. J. Zverko, J. Ziznovsky, S. J. Adelman, \& W. W. Weiss, IAU Symp., 224, 786

Mantegazza, L. 2000, in Delta Scuti and Related Stars, ed. M. Breger, \& M. Montgomery, PASPC, 210, 138

Mathias, P., \& Aerts, C. 1996, A\&A, 312, 905

Østensen, R. H. 2009, Commun. Asteroseismol., 159, in press

Østensen, R. H., Telting, J. H., Heber, U., \& Jeffery, C. S. 2008, in Hot Subdwarf Stars and Related Objects, ed. U. Heber, C. S. Jeffery, \& R. Napiwotzki, PASPC, 392, 301

O'Toole, S. J., \& Heber, U. 2006, A\&A, 452, 579

O'Toole, S. J., Bedding, T. R., Kjeldsen, H., et al. 2000, ApJ, 537, L53

O’Toole, S. J., Bedding, T. R., Kjeldsen, H., Dall, T. H., \& Stello, D. 2002, MNRAS, 334, 471

O’Toole, S. J., Jørgensen, M. S., Kjeldsen, H., et al. 2003a, MNRAS, 340, 856 O'Toole, S. J., Jørgensen, M. S., Kjeldsen, H., et al. 2003b, MNRAS, 340, 856

O'Toole, S. J., Heber, U., Jeffery, C. S., et al. 2005, A\&A, 440, 667

Reed, M. D., Kilkenny, D., Kawaler, S. D., et al. 2000, Baltic Astron., 9, 183

Reed, M. D., \& Whole Earth Telescope Xcov 21 and 23 Collaborations 2006, Mem. Soc. Astron. Ital., 77, 417

Reed, M. D., Eggen, J. R., Harms, S. L., et al. 2009, A\&A, 493, 175

Schrijvers, C., Telting, J. H., Aerts, C., Ruymaekers, E., \& Henrichs, H. F. 1997, A\&AS, 121, 343

Telting, J. H. \& Østensen, R. H. 2004, A\&A, 419, 685

Telting, J. H. \& Østensen, R. H. 2006, A\&A, 450, 1149

Telting, J., Geier, S., Østensen, R., et al. 2008, A\&A, 492, 815

Tillich, A., Heber, U., O’Toole, S. J., Østensen, R., \& Schuh, S. 2007, A\&A, 473, 219

Townsend, R. H. D. 1997, MNRAS, 284, 839

Vučković, M., Aerts, C., Østensen, R., et al. 2007, A\&A, 471, 605

Woolf, V. M., Jeffery, C. S., \& Pollacco, D. L. 2002, MNRAS, 329, 497

Zima, W. 2006, A\&A, 455, 227

Zima, W. 2008, Commun. Asteroseismol., 155, 17 